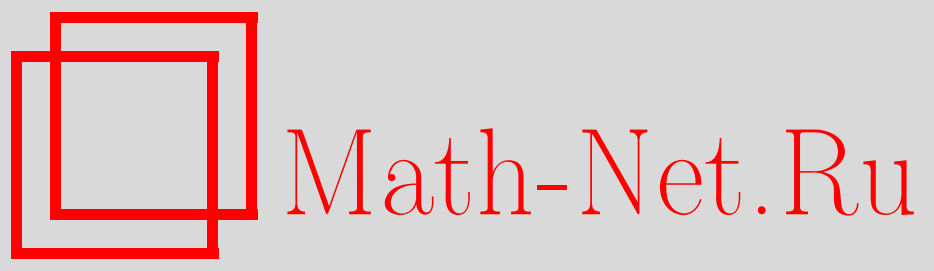

В. Г. Михайлов, А. М. Шойтов, О длинных повторениях цепочек в цепи Маркова, Дискрет. матем., 2014, том 26, выпуск 3, 79-89

DOI: https://doi.org/10.4213/dm1292

Использование Общероссийского математического портала Math-Net.Ru подразумевает, что вы прочитали и согласны с пользовательским соглашением http://www . mathnet.ru/rus/agreement

Параметры загрузки:

IP: 52.23 .180 .231

26 апреля 2023 г., 14:49:12

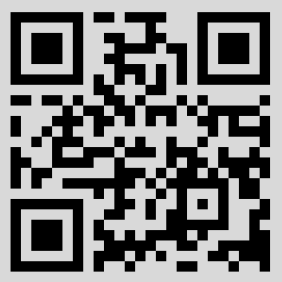




\title{
О длинных повторениях цепочек в цепи Маркова
}

\author{
() 2014 г. В. Г. Михайлов*, А. М. Шойтов**
}

Пусть $X_{0}, X_{1}, \ldots$ - простая эргодическая цепь Маркова с конечным числом состояний. Доказываются предельные теоремы для числа $\tilde{\xi}(s, n)$ событий

$$
\left\{X_{i-1} \neq X_{j-1}, X_{i+k}=X_{j+k}, k=0, \ldots, s-1\right\}, \quad 1 \leqslant i<j \leqslant n,
$$

когда $s, n \rightarrow \infty$. Из них выводятся предельные теоремы для некоторых связанных с $\tilde{\xi}(s, n)$ случайных величин.

Ключевые слова: цепь Маркова, повторения $s$-цепочек, предельная теорема Пуассона.

\section{1. Введение}

Пусть последовательность $\mathbf{X}=\left\{X_{0}, X_{1}, \ldots\right\}$ образует простую однородную неразложимую и ациклическую цепь Маркова с состояниями $1, \ldots, N$, матрицей переходных вероятностей $\mathbf{P}=\left\|p_{a, b}\right\|$ и произвольным начальным распределением. Отметим, что такая цепь является эргодической.

Положим $Y_{i}(s)=\left(X_{i}, \ldots, X_{i+s-1}\right), s, i=1,2, \ldots$, и рассмотрим события $\left\{Y_{i_{1}}(s)=\right.$ $\left.Y_{i_{2}}(s)\right\}$ и $\left\{X_{i_{1}-1} \neq X_{i_{2}-1}, Y_{i_{1}}(s)=Y_{i_{2}}(s)\right\}$. Первое событие состоит в совпадении указанных цепочек (в [1] для него был использован термин «повторение цепочки», ему мы и следуем в заглавии статьи), а второе заключается в том, что в моменты $i_{1}$ и $i_{2}$ начинаются совпадающие между собой отрезки последовательности длины не меньше $s$. Это событие можно трактовать как начало серии повторений $s$-цепочек. Каждая такая серия имеет, вообще говоря, свою случайную длину. Пусть

$$
\xi_{n}(s)=\sum_{1 \leqslant i_{1}<i_{2} \leqslant n} I\left\{Y_{i_{1}}(s)=Y_{i_{2}}(s)\right\}
$$

- число совпадений $s$-цепочек, а

$$
\tilde{\xi}_{n}(s)=\sum_{1 \leqslant i_{1}<i_{2} \leqslant n} I\left\{X_{i_{1}-1} \neq X_{i_{2}-1}, Y_{i_{1}}(s)=Y_{i_{2}}(s)\right\}
$$

* Место работы: Математический институт им. В.А. Стеклова РАН, e-mail: mikhail@mi.ras.ru

** Место работы: Академия криптографии Российской Федерации, e-mail: shamru1@yandex.ru 
- число серий повторений $s$-цепочек, все они начинаются в период с момента 1 до момента $n$ включительно.

В [1] эти величины рассматривались в последовательности полиномиальных испытаний, и было, в частности, показано, что при $s, n \rightarrow \infty$ и в области пуассоновского предела распределения этих величин сходятся к сложному пуассоновскому и простому пуассоновскому распределениям. Некоторые свойства последовательности $\left\{Y_{1}(s), Y_{2}(s), \ldots\right\}$ исследовались в работах П.Ф. Беляева (см., например, [2]).

В настоящей работе изучается распределение величины $\tilde{\xi}_{n}(s)$ в неразложимой и ациклической марковской цепи. Выводятся достаточные условия сходимости (при $n, s \rightarrow \infty)$ распределения случайной величины $\tilde{\xi}_{n}(s)$ к распределению Пуассона и к нормальному распределению. При этом распределение цепи $\mathbf{X}$ считается фиксированным. В качестве следствий этих предельных теорем получены предельные теоремы для времени первого повторения $s$-цепочек $\tau(s)$ и для максимальной длины повторившейся цепочки $\mu(n)$. Эти величины определяются формулами

$$
\tau(s)=\min \left\{n: \tilde{\xi}_{n}(s)>0\right\}, \quad \mu(n)=\max \left\{s: \tilde{\xi}_{n}(s)>0\right\} .
$$

Аналогичные задачи для цепочек в последовательности независимых полиномиальных испытаний были решены в [1] и [3]. В [4] приведен обзор результатов по повторениям цепочек в независимых дискретных последовательностях с точностью до отношения эквивалентности. Задачи, связанные с совпадениями цепочек из двух независимых конечных цепей Маркова, рассматривались в [5]. В [6] были изучены условия сходимости к сложному распределению Пуассона распределения числа серий в конечной цепи Маркова. Задачи о повторениях состояний и цепочек состояний заданной длины в цепях Маркова в схемах с возрастающим числом исходов рассматривались в [7], [8], [9].

\section{2. Формулировки}

Пусть $p=\max _{a, b} p_{a, b}, \pi=\left(\pi_{1}, \ldots, \pi_{N}\right), \pi_{k}>0, k=1, \ldots, N$, - стационарное распределение цепи $\mathbf{X}, \Pi_{2}=\sum_{k=1}^{N} \pi_{k}^{2}$.

Рассмотрим матрицу $\mathbf{P}_{\mathbf{2}}=\left\|p_{a, b}^{2}\right\|$. Матрица $\mathbf{P}_{\mathbf{2}}$ является неразложимой и примитивной (см. [10]), так как расположение нулевых элементов в ней совпадает с расположением нулевых элементов в матрице $\mathbf{P}$. Пусть $\rho>0-$ максимальное характеристическое число матрицы $\mathbf{P}_{\mathbf{2}}, z=\left(z_{1}, \ldots, z_{N}\right), z_{k}>0, k=1, \ldots, N$, - соответствующий ему собственный вектор: $\mathbf{P}_{\mathbf{2}} z=\rho z$. Согласно предложению 2 на с. 365 книги [10] для $\mathbf{P}_{\mathbf{2}}$ имеет место представление

$$
\mathbf{P}_{2}=Z \rho \mathbf{P}_{2}^{\prime} Z^{-1}
$$

где $Z$ - диагональная матрица, на главной диагонали которой расположены числа $\left(z_{1}, \ldots, z_{N}\right)$, а $\mathbf{P}_{\mathbf{2}}^{\prime}-$ стохастическая матрица.

Матрица $\mathbf{P}_{2}^{\prime}$ определяет неразложимую и ациклическую цепь Маркова с некоторым стационарным распределением $\pi^{\prime}=\left(\pi_{1}^{\prime}, \ldots, \pi_{N}^{\prime}\right), \pi_{k}^{\prime}>0, k=1, \ldots, N$, и для некоторой константы $c_{1}>0$ при $s \rightarrow \infty$ справедлива оценка

$$
\left|\mathbf{P}_{\mathbf{2}}^{\prime s}-\boldsymbol{\Pi}^{\prime}\right|=O\left(e^{-c_{1} s}\right),
$$


где матрица $\boldsymbol{\Pi}^{\prime}$ состоит из одинаковых строк $\pi^{\prime}$, а $|A|-$ норма матрицы $A=\left\|a_{i, j}\right\|$, определяемая формулой (см. [10])

$$
|A|=\max _{1 \leqslant i \leqslant N} \sum_{j=1}^{N}\left|a_{i, j}\right| .
$$

Введем обозначения $\mathcal{L}(W)$ для распределения случайной величины $W$ и $\operatorname{Po}(\lambda)$ для распределения Пуассона с параметром $\lambda$, а также обозначения

$$
\begin{gathered}
\left(\pi^{\prime}, z^{-1}\right)=\sum_{k=1}^{N} \pi_{k}^{\prime} z_{k}^{-1}, \quad\left(\pi^{2}, z\right)=\sum_{l=1}^{N} \pi_{l}^{2} z_{l}, \\
R=\sqrt{\rho^{s-1}(1-\rho)\left(\pi^{\prime}, z^{-1}\right)\left(\pi^{2}, z\right)}, \quad \Lambda_{s, n}=\frac{n^{2}}{2} R^{2} .
\end{gathered}
$$

Пусть $\rho(\Psi, \Gamma)$ - расстояние по вариации между распределениями $\Psi$ и $Г$.

Теорема 1. Пусть $p^{2}<\rho, n, s \rightarrow \infty$ и выполнено условие $\Lambda_{s, n} \rightarrow \lambda \in(0, \infty)$. Тогда $\mathcal{L}\left(\tilde{\xi}_{n}(s)\right) \rightarrow \operatorname{Po}(\lambda)$, причем

$$
\rho\left(\mathcal{L}\left(\tilde{\xi}_{n}(s)\right), \operatorname{Po}(\lambda)\right)=O\left(e^{-c s}+\left|\Lambda_{s, n}-\lambda\right|\right),
$$

для любого $c, 0<c<\min \left\{1, c_{1}, c_{2}, c_{3}\right\}$, где $c_{1}$ определено в $(1), c_{2}=\frac{1}{2} \ln \left(\frac{\rho}{p^{2}}\right), a$ $c_{3}=\frac{1}{2} \ln (1 / \rho)$.

Теорема 2. Пусть $p^{2}<\rho, n, s \rightarrow \infty$ и выполнено условие

$$
\Lambda_{s, n} \rightarrow \infty, \quad \Lambda_{s, n}=O\left(e^{c s}\right)
$$

где $0<c<2 c_{2}$. Тогда функиия распределения случайной величины $\Lambda_{s, n}^{-1 / 2}\left(\tilde{\xi}_{n}(s)-\right.$ $\left.\mathbf{E} \tilde{\xi}_{n}(s)\right)$ сходится $к$ стандартной нормальной функиии распределения.

Замечание 1. Условие $p^{2}<\rho$ появилось в теоремах 1 и 2 как техническое. Можно показать, что если в матрице переходных вероятностей $\mathbf{P}$ максимальная вероятность лежит на главной диагонали (т. е. $p=p_{a, a}$ при некотором $a$ ) и $p<1$, то условие $p^{2}<\rho$ выполнено. Вопрос о выполнении этого условия в общем случае требует отдельного изучения. Отметим только, что случае выполнения неравенства $p^{2} \geqslant \rho$ предельное распределение Пуассона в теореме 1 сменилось бы сложным пуассоновским распределением.

Замечание 2. Пусть строки матрицы $\mathbf{P}$ являются перестановками одного и того же фиксированного вектора. Тогда $\rho=P_{2}$, где $P_{2}=\sum_{i=1}^{N} p_{i}^{2}, p<\sqrt{P}_{2}$, вектор $\left(z_{1}, \ldots, z_{N}\right)=(1, \ldots, 1)$, a $\Lambda_{s, n}=\frac{n^{2}}{2} P_{2}^{s-1}\left(1-P_{2}\right) \Pi_{2}$.

Теорема 3. Пусть $p^{2}<\rho, a s \rightarrow \infty$. Тогда

$$
\mathbf{P}\{\tau(s) R \leqslant x\}=1-e^{-x^{2} / 2}+O\left(e^{-c s}\right) \quad \forall x>0
$$

при любом $c, 0<c<\min \left\{1, c_{1}, c_{2}, c_{3}\right\}$. 
Теорема 4. Пусть $p^{2}<\rho, a n \rightarrow \infty$. Тогда

$$
\mathbf{P}\left\{\mu(n)-\left[\frac{2 \ln n}{|\ln \rho|}\right]<k\right\} \rightarrow \exp \left(-\frac{1-\rho}{2} \rho^{k-\{2 \ln n /|\ln \rho|\}}\right),
$$

где $[u] и\{u\}-$ целая и дробная части числа $и$.

При выводе этих теорем используется следующая оценка точности пуассоновской аппроксимации для распределения величины $\tilde{\xi}(s, n)$.

Введем множество

$$
J_{s, n}=\left\{\bar{i}=\left(i_{1}, i_{2}\right): s^{3}+1 \leqslant i_{1}<i_{1}+s^{3}<i_{2} \leqslant n\right\}
$$

и определим случайные величины

$$
\delta_{n}(s)=\sum_{\left(i_{1}, i_{2}\right) \in J_{s, n}} I\left\{X_{i_{1}-1} \neq X_{i_{2}-1}, Y_{i_{1}}(s)=Y_{i_{2}}(s)\right\} .
$$

Замечание 3. В определении множества $J_{s, n}$ вместо величины $s^{3}$ может быть использована любая достаточно быстро растущая при $s \rightarrow \infty$ функция. От выбора этой функции зависят лишь конкретный вид оценки приводимой далее теоремы 5 и границы для констант в оценках теорем 1 и 3. При нашем выборе упомянутые границы вычисляются просто.

Теорема 5. Пусть $s, n \rightarrow \infty, s^{3}<n$. Тогда

$$
\begin{gathered}
\rho\left(\mathcal{L}\left(\tilde{\xi}_{n}(s)\right), \operatorname{Po}\left(\mathbf{E} \delta_{n}(s)\right)\right)= \\
=O\left(s^{2} n^{-1} \mathbf{E} \delta_{n}(s)+s^{3} n p^{s}+\min \left(\mathbf{E} \delta_{n}(s), \sqrt{\mathbf{E} \delta_{n}(s)}\right) e^{-c s^{2}}\right)
\end{gathered}
$$

при некотором с $>0$.

\section{3. Доказательства}

Доказательство. Доказательство теоремы 5 состоит из трех частей. Сначала оценивается величина $\rho\left(\mathcal{L}\left(\tilde{\xi}_{n}(s)\right), \mathcal{L}\left(\delta_{n}(s)\right)\right)$, затем - величина $\rho\left(\mathcal{L}\left(\delta_{n}(s)\right), \operatorname{Po}\left(\mathbf{E} \delta_{n}(s)\right)\right)$. На заключительном шаге остается применить неравенство треугольника.

1. Используя неравенство $\mathbf{P}\left\{X_{i_{2}}=x_{2} \mid X_{i_{1}}=x_{1}\right\} \leqslant p$ при $i_{2} \geqslant i_{1}+s$, получаем оценки

$$
\begin{gathered}
\mathbf{P}\left\{Y_{i_{1}}(s)=Y_{i_{2}}(s)\right\}= \\
=\sum_{\left(x_{1}, \ldots, x_{s}\right) \in\{1, \ldots, N\}^{s}} \mathbf{P}\left\{X_{i_{1}}=x_{1}\right\} \mathbf{P}\left\{X_{i_{2}}=x_{1} \mid X_{i_{1}+s-1}=x_{s}\right\} \prod_{k=2}^{s} p_{x_{k-1}, x_{k}}^{2} \leqslant \\
\leqslant \sum_{\left(x_{1}, \ldots, x_{s}\right) \in\{1, \ldots, N\}^{s}} \mathbf{P}\left\{X_{i_{1}}=x_{1}\right\} p^{s} p_{x_{1}, x_{2}} p_{x_{2}, x_{3}} \cdot \ldots \cdot p_{x_{s-1}, x_{s}}=p^{s} .
\end{gathered}
$$

Далее по аналогии с [1] и [11] заметим, что при $\left(i_{2}=i_{1}+1, \ldots, i_{1}+s-1\right)$ фиксация вектора $Y_{i_{1}}\left(i_{2}-i_{1}\right)=\left(x_{1}, \ldots, x_{i_{2}-i_{1}}\right)$ однозначно определяет все компоненты вектора $Y_{i_{2}}(s)=\left(x_{1}, \ldots, x_{i_{2}-i_{1}}, x_{1}, \ldots\right)$. Поэтому

$$
\mathbf{P}\left\{Y_{i_{1}}(s)=Y_{i_{2}}(s)\right\}=
$$




$$
\begin{gathered}
=\sum_{\left(x_{1}, \ldots, x_{i_{2}-i_{1}}\right) \in\{1, \ldots, N\}^{i_{2}-i_{1}}} \mathbf{P}\left\{Y_{i_{1}}\left(i_{2}-i_{1}\right)=\left(x_{1}, \ldots, x_{i_{2}-i_{1}}\right)\right\} \times \\
\times p_{x_{1}, x_{2}} p_{x_{2}, x_{3}} \cdot \ldots \cdot p_{x_{i_{2}-i_{1}-1}, x_{i_{2}-i_{1}}} p_{x_{i_{2}-i_{1}}, x_{1}} \cdot \ldots \leqslant p^{s} .
\end{gathered}
$$

Теперь, пользуясь неравенством $\tilde{\xi}_{n}(s) \geqslant \delta_{n}(s)$, получаем

$$
\begin{gathered}
\rho\left(\mathcal{L}\left(\tilde{\xi}_{n}(s)\right), \mathcal{L}\left(\delta_{n}(s)\right)\right) \leqslant \mathbf{E}\left(\tilde{\xi}_{n}(s)-\delta_{n}(s)\right) \leqslant \\
\leqslant \sum_{i_{1}=2}^{s^{3}} \sum_{i_{2}=i_{1}+1}^{n} \mathbf{P}\left\{X_{i_{1}-1} \neq X_{i_{2}-1}, Y_{i_{1}}(s)=Y_{i_{2}}(s)\right\}+ \\
+\sum_{i_{1}=2}^{n-1} \sum_{i_{2}=i_{1}+1}^{i_{1}+s^{3}} \mathbf{P}\left\{X_{i_{1}-1} \neq X_{i_{2}-1}, Y_{i_{1}}(s)=Y_{i_{2}}(s)\right\} \leqslant 2 s^{3} n p^{s} .
\end{gathered}
$$

2. Сначала оценим $\mathbf{E} \delta_{n}(s)$. Для $\left(i_{1}, i_{2}\right) \in J_{s, n}$ имеем

$$
\begin{gathered}
\mathbf{P}\left\{Y_{i_{1}}(s)=Y_{i_{2}}(s)\right\}= \\
=\sum_{\left(x_{1}, x_{2}, \ldots, x_{s}\right) \in\{1, \ldots, N\}^{s}} \mathbf{P}\left\{Y_{i_{1}}(s)=\left(x_{1}, x_{2}, \ldots, x_{s}\right)\right\} \times \\
\times \mathbf{P}\left\{Y_{i_{2}}(s)=\left(x_{1}, x_{2}, \ldots, x_{s}\right) \mid Y_{i_{1}}(s)=\left(x_{1}, x_{2}, \ldots, x_{s}\right)\right\}= \\
=\sum_{\left(x_{1}, x_{2}, \ldots, x_{s}\right) \in\{1, \ldots, N\}^{s}} \mathbf{P}\left\{X_{i_{1}}=x_{1}\right\} p_{x_{1}, x_{2}} p_{x_{2}, x_{3}} \cdot \ldots \cdot p_{x_{s-1}, x_{s}} \times \\
\times \mathbf{P}\left\{Y_{i_{2}}(s)=\left(x_{1}, x_{2}, \ldots, x_{s}\right) \mid X_{i_{1}+s-1}=x_{s}\right\}= \\
\sum_{\left(x_{1}, x_{2}, \ldots, x_{s}\right) \in\{1, \ldots, N\}^{s}} \mathbf{P}\left\{X_{i_{1}}=x_{1}\right\} \cdot p_{x_{s}, x_{1}}^{\left(i_{2}-i_{1}-s-1\right)} \cdot p_{x_{1}, x_{2}}^{2} \cdot p_{x_{2}, x_{3}}^{2} \cdot \ldots \cdot p_{x_{s-1}, x_{s}}^{2} .
\end{gathered}
$$

Так как для $\left(i_{1}, i_{2}\right) \in J_{s, n}$ справедливы оценки $i_{2}-i_{1}>s^{3}, i_{1}>s^{3}$, а цепь Маркова $\mathbf{X}$ является эргодической, то для некоторой константы $c_{4}>0$, которую при необходимости можно явно выписать, равномерно по $\left(i_{1}, i_{2}\right) \in J_{s, n}$ и $x_{1}, x_{s} \in$ $\{1, \ldots, N\}$ справедливы оценки

$$
\left|p_{x_{s}, x_{1}}^{\left(i_{2}-i_{1}-s-1\right)}-\pi_{x_{1}}\right|<e^{-c_{4} s^{3}}, \quad\left|\mathbf{P}\left\{X_{i_{1}}=x_{1}\right\}-\pi_{x_{1}}\right|<e^{-c_{4} s^{3}} .
$$

Поэтому равномерно по $\left(i_{1}, i_{2}\right) \in J_{s, n}$

$$
\mathbf{P}\left\{Y_{i_{1}}(s)=Y_{i_{2}}(s)\right\}=\sum_{\left(x_{1}, x_{2}, \ldots, x_{s}\right) \in\{1, \ldots, N\}^{s}} \pi_{x_{1}}^{2} \cdot p_{x_{1}, x_{2}}^{2} \cdot p_{x_{2}, x_{3}}^{2} \cdot \ldots \cdot p_{x_{s-1}, x_{s}}^{2}\left(1+O\left(e^{-c_{4} s^{3}}\right)\right) .
$$

По аналогии с (11) в векторном виде, используя также (3) и соотношение $e^{-c_{4} s^{3}}=$ $O\left(e^{-c_{1} s}\right)$ при $s \rightarrow \infty$, получаем

$$
\left(\mathbf{P}\left\{Y_{i_{1}}(s)=Y_{i_{2}}(s), X_{i_{1}+s-1}=a\right\}, a=1, \ldots, N\right)=
$$




$$
\begin{gathered}
=\left(\pi_{k}^{2}\left(1+O\left(e^{-c_{4} s^{3}}\right)\right), k=1, \ldots, N\right) \times \mathbf{P}_{\mathbf{2}}{ }^{s-1}= \\
=\left(\pi_{k}^{2}\left(1+O\left(e^{-c_{4} s^{3}}\right)\right), k=1, \ldots, N\right) \times\left(Z \rho \mathbf{P}_{\mathbf{2}}^{\prime} Z^{-1}\right)^{s-1}= \\
=\rho^{s-1}\left(1+O\left(e^{-c_{4} s^{3}}\right)\right)\left(\pi_{k}^{2}, k=1, \ldots, N\right) \times Z \mathbf{P}_{\mathbf{2}}^{\prime s-1} Z^{-1}= \\
=\rho^{s-1}\left(1+O\left(e^{-c_{1} s}\right)\right)\left(\pi_{k}^{2}, k=1, \ldots, N\right) \times Z \mathbf{\Pi}^{\prime} Z^{-1}= \\
=\rho^{s-1}\left(1+O\left(e^{-c_{1} s}\right)\right)\left(\pi_{k}^{\prime} z_{k}^{-1} \sum_{l=1}^{N} \pi_{l}^{2} z_{l}, k=1, \ldots, N\right) .
\end{gathered}
$$

Так как

$$
\mathbf{P}\left\{Y_{i_{1}}(s)=Y_{i_{2}}(s)\right\}=\sum_{a=1}^{N} \mathbf{P}\left\{Y_{i_{1}}(s)=Y_{i_{2}}(s), X_{i_{1}+s-1}=a\right\},
$$

то

$$
\mathbf{P}\left\{Y_{i_{1}}(s)=Y_{i_{2}}(s)\right\}=\rho^{s-1} \sum_{k=1}^{N} \pi_{k}^{\prime} z_{k}^{-1} \sum_{l=1}^{N} \pi_{l}^{2} z_{l} \cdot\left(1+O\left(e^{-c_{1} s}\right)\right),
$$

откуда, используя равенство

$$
\begin{gathered}
\mathbf{P}\left\{X_{i_{1}-1} \neq X_{i_{2}-1}, Y_{i_{1}}(s)=Y_{i_{2}}(s)\right\}= \\
=\mathbf{P}\left\{Y_{i_{1}}(s)=Y_{i_{2}}(s)\right\}-\mathbf{P}\left\{Y_{i_{1}-1}(s+1)=Y_{i_{2}-1}(s+1)\right\},
\end{gathered}
$$

получаем (в обозначениях (1)), что равномерно по $\left(i_{1}, i_{2}\right) \in J_{s, n}$

$$
\begin{gathered}
\mathbf{P}\left\{X_{i_{1}-1} \neq X_{i_{2}-1}, Y_{i_{1}}(s)=Y_{i_{2}}(s)\right\}= \\
=\rho^{s-1}(1-\rho)\left(\pi^{\prime}, z^{-1}\right)\left(\pi^{2}, z\right)\left(1+O\left(e^{-c_{1} s}\right)\right) .
\end{gathered}
$$

Следовательно (опять см. (1)),

$$
\mathbf{E} \delta_{n}(s)=\frac{C_{n-2 s^{3}}^{2}}{n^{2} / 2} \Lambda_{s, n}\left(1+O\left(e^{-c_{1} s}\right)\right)=\Lambda_{s, n}\left(1+O\left(\frac{s^{3}}{n}+e^{-c_{1} s}\right)\right) .
$$

Оценим точность аппроксимации распределения случайной величины $\delta_{n}(s)$ распределением Пуассона с параметром $\mathbf{E} \delta_{n}(s)$. Для этого представим случайную величину $\delta_{n}(s)$ в виде суммы случайных индикаторов:

$$
\delta_{n}(s)=\sum_{\bar{i} \in J_{s, n}} \eta(\bar{i}),
$$

где $\bar{j}=\left(j_{1}, j_{2}\right), \eta(\bar{i})=I\left\{X_{i_{1}-1} \neq X_{i_{2}-1}, Y_{i_{1}}(s)=Y_{i_{2}}(s)\right\}$.

Для исследования распределения этой суммы воспользуемся методом Чена Стейна (см. [12]). Следуя этому методу, для любой пары $\bar{i}=\left(i_{1}, i_{2}\right)$ из $J_{s, n}$ определим множество $\Gamma(\bar{i})$ таких пар $\bar{j}=\left(j_{1}, j_{2}\right)$ из $J_{s, n}, \quad \bar{j} \neq \bar{i}$, что хотя бы один из 
отрезков $\left[i_{1}-1, i_{1}+s-1\right],\left[i_{2}-1, i_{2}+n-1\right]$ пересекается хотя бы с одним из отрезков $\left[j_{1}-1, j_{1}+n-1\right],\left[j_{2}-1, j_{2}+n-1\right]$ или лежит на расстоянии менее $s^{2}$ от него.

Для $\bar{i}=\left(i_{1}, i_{2}\right)$ из $J_{s, n}$ положим $\eta(\bar{i})=I\left\{Y_{i_{1}}(s)=Y_{i_{2}}(s)\right\}$,

$$
\begin{gathered}
U_{\bar{i}}=\sum_{\bar{j} \in \Gamma(\bar{i})} I\left\{X_{i_{1}-1} \neq X_{i_{2}-1}, Y_{i_{1}}(s)=Y_{i_{2}}(s)\right\}, \\
V_{\bar{i}}=\sum_{\bar{j} \in J_{s, n} \backslash \Gamma(\bar{i})} I\left\{X_{i_{1}-1} \neq X_{i_{2}-1}, Y_{i_{1}}(s)=Y_{i_{2}}(s)\right\} .
\end{gathered}
$$

Согласно теореме 2.N работы [12] в нашем случае справедлива следующая верхняя оценка

$$
\begin{aligned}
& \rho\left(\mathcal{L}\left(\delta_{n}(s)\right), \operatorname{Po}\left(\mathbf{E} \delta_{n}(s)\right)\right) \leqslant \frac{1-e^{-\mathbf{E} \delta_{n}(s)}}{\mathbf{E} \delta_{n}(s)}\left(\sum_{\bar{i} \in J_{s, n}}(\mathbf{E} \eta(\bar{i})(\mathbf{E} \eta(\bar{i})+\right. \\
& \left.\left.\left.\quad+\sum_{\bar{j} \in \Gamma(\bar{i})} \mathbf{E} \eta(\bar{j})\right)\right)+\sum_{\bar{i} \in J_{s, n}} \sum_{\bar{j} \in \Gamma(\bar{i})} \mathbf{E}\left(\eta(\bar{j}) \eta^{\prime}(\bar{i})\right)\right)+ \\
& \left.+\min \left(1, \sqrt{\frac{2}{e \mathbf{E} \delta_{n}(s)}}\right) \sum_{\bar{i} \in J_{s, n}} \mathbf{E} \mid \mathbf{E} \eta(\bar{i})-\mathbf{E}\left\{\eta(\bar{i}) \mid V_{\bar{i}}\right\}\right) \mid .
\end{aligned}
$$

Учитывая, что $|\Gamma(\bar{i})| \leqslant 4 n(s+1)^{2}$ для всех $\bar{i} \in J_{s, n}$, сразу получаем

$$
\sum_{\bar{i} \in J_{s, n}}\left(\mathbf{E} \eta(\bar{i})\left(\mathbf{E} \eta(\bar{i})+\sum_{\bar{j} \in \Gamma(\bar{i})} \mathbf{E} \eta(\bar{j})\right)\right)=O\left(\left(\mathbf{E} \delta_{n}(s)\right)^{2} s^{2} n^{-1}\right) .
$$

Далее для любой пары $\bar{i}=\left(i_{1}, i_{2}\right)$ из $J_{s, n}$ разобьем множество $\Gamma(\bar{i})$ на пять подмножеств:

$\Gamma_{1}(\bar{i})-$ ровно один из отрезков $\left[j_{1}-1, j_{1}+s-1\right],\left[j_{2}-1, j_{2}+s-1\right]$ пересекается с одним из отрезков $\left[i_{1}, i_{1}+s-1\right],\left[i_{2}, i_{2}+s-1\right]$, а остальные отрезки лежат на расстоянии не менее $s^{2}$ друг от друга.

$\Gamma_{1}^{\prime}(\bar{i})-$ ровно один из отрезков $\left[j_{1}-1, j_{1}+s-1\right],\left[j_{2}-1, j_{2}+s-1\right]$ лежит на расстоянии менее $s^{2}$ от одного из отрезков $\left[i_{1}, i_{1}+s-1\right],\left[i_{2}, i_{2}+s-1\right]$, но не пересекается с ним.

$\Gamma_{2}(\bar{i})$ - оба отрезка $\left[j_{1}-1, j_{1}+s-1\right],\left[i_{2}-1, i_{2}+s-1\right]$ пересекаются с обоими отрезками $\left[i_{1}-1, i_{1}+s-1\right],\left[i_{2}-1, i_{2}+s-1\right]$ (при этом минимальное расстояние между непересекающимися отрезками более $\left.s^{2}-2 s\right)$.

$\Gamma_{2}^{\prime}(\bar{i})$ - один из отрезков $\left[j_{1}-1, j_{1}+s-1\right],\left[j_{2}-1, j_{2}+s-1\right]$ пересекается с одним из отрезков $\left[i_{1}-1, i_{1}+s-1\right],\left[i_{2}-1, i_{2}+s-1\right]$, а другой лежит на расстоянии менее $s^{2}$ от оставшегося, но с ним не пересекается.

$\Gamma_{2}^{\prime \prime}(\bar{i})$ - оба отрезка $\left[j_{1}-1, j_{1}+s-1\right],\left[j_{2}-1, j_{2}+s-1\right]$ лежат на расстоянии менее $s^{2}$ от отрезков $\left[i_{1}-1, i_{1}+s-1\right],\left[i_{2}-1, i_{2}+s-1\right]$, но с ними не пересекаются.

По аналогии с рассуждениями в [1] и [11] с учетом $(3)$ и неравенства $\eta(\bar{i}) \leqslant$ $I\left\{Y_{i_{1}}(s)=Y_{i_{2}}(s)\right\}$ получаем оценки

$$
\sum_{\bar{i} \in J_{s, n}} \sum_{\bar{j} \in \Gamma_{2}^{\prime}(\bar{i})} \mathbf{E}(\eta(\bar{j}) \eta(\bar{i})) \leqslant
$$




$$
\begin{aligned}
& \leqslant 8(s+1)^{3} p^{s-1} \sum_{\bar{i} \in J_{s, n}} \mathbf{P}(\eta(\bar{i}))=O\left(\mathbf{E} \delta_{n}(s) s^{3} p^{s-1}\right), \\
& \sum_{\bar{i} \in J_{s, n}} \sum_{\bar{j} \in \Gamma_{1}^{\prime}(\bar{i})} \mathbf{E}(\eta(\bar{j}) \eta(\bar{i})) \leqslant \\
& \leqslant 4(s+1)^{2} n p^{s-1} \sum_{\bar{i} \in J_{s, n}} \mathbf{E}(\eta(\bar{i}))=O\left(\mathbf{E} \delta_{n}(s) s^{2} n p^{s-1}\right), \\
& \sum_{\bar{i} \in J_{s, n}} \sum_{\bar{j} \in \Gamma_{2}^{\prime \prime}(\bar{i})} \mathbf{E}(\eta(\bar{j}) \eta(\bar{i})) \leqslant \\
& \leqslant 16(s+1)^{4} p^{s-1} \sum_{\bar{i} \in J_{s, n}} \mathbf{E}(\eta(\bar{i}))=O\left(\mathbf{E} \delta_{n}(s) s^{4} p^{s-1}\right), \\
& \sum_{\bar{i} \in J_{s, n}} \sum_{\bar{j} \in \Gamma_{1}(\bar{i})} \mathbf{E}(\eta(\bar{j}) \eta(\bar{i})) \leqslant \\
& \leqslant 4(s+1) n p^{s-1} \sum_{\bar{i} \in J_{s, n}} \mathbf{E}(\eta(\bar{i}))=O\left(\mathbf{E} \delta_{n}(s) s n p^{s-1}\right), \\
& \sum_{\bar{i} \in J_{s, n}} \sum_{\bar{j} \in \Gamma_{2}(\bar{i})} \mathbf{E}(\eta(\bar{j}) \eta(\bar{i})) \leqslant \\
& \sum_{s+1}^{2} n^{2} p^{2(s-1)}=O\left(\left(s n p^{s-1}\right)^{2}\right) .
\end{aligned}
$$

Далее оценим величину

$$
\left.\sum_{\bar{i} \in J_{s, n}} \mathbf{E} \mid \mathbf{E} \eta^{\prime}(\bar{i})-\mathbf{E}\left\{\eta^{\prime}(\bar{i}) \mid V_{\bar{i}}\right\}\right) \mid .
$$

С учетом марковского свойства и эргодичности цепи $\mathbf{X}$ имеем

$$
\begin{gathered}
\mathbf{E}\left|\mathbf{E} \eta(\bar{i})-\mathbf{E}\left\{\eta(\bar{i}) \mid V_{\bar{i}}\right\}\right| \leqslant \\
\leqslant \mathbf{E}\left|\mathbf{E} \eta(\bar{i})-\mathbf{E}\left\{\eta(\bar{i}) \mid X_{1}, \ldots, X_{i_{1}-s^{2}}, X_{i_{1}+s+s^{2}}, \ldots, X_{i_{2}-s^{2}}, X_{i_{2}+s+s^{2}}, \ldots\right\}\right|= \\
=\mathbf{E}\left|\mathbf{E} \eta(\bar{i})-\mathbf{E}\left\{\eta(\bar{i}) \mid X_{i_{1}-s^{2}}, X_{i_{1}+s+s^{2}}, X_{i_{2}-s^{2}}, X_{i_{2}+s+s^{2}}\right\}\right| \leqslant \\
\leqslant \mathbf{E}\left|\mathbf{E} \eta(\bar{i})-\mathbf{E}\left\{\eta(\bar{i}) \mid X_{i_{2}+s+s^{2}}\right\}\right|+ \\
+\mathbf{E}\left|\mathbf{E}\left\{\eta(\bar{i}) \mid X_{i_{2}+s+s^{2}}\right\}-\mathbf{E}\left\{\eta^{\prime}(\bar{i}) \mid X_{i_{2}-s^{2}}, X_{i_{1}+s+s^{2}}\right\}\right|+ \\
+\mathbf{E}\left|\mathbf{E}\left\{\eta(\bar{i}) \mid X_{i_{2}-s^{2}}, X_{i_{1}+s+s^{2}}\right\}-\mathbf{E}\left\{\eta^{\prime}(\bar{i}) \mid X_{i_{1}+s+s^{2}}, X_{i_{2}-s^{2}}, X_{i_{2}+s+s^{2}}\right\}\right|+ \\
+\mathbf{E} \mid \mathbf{E}\left\{\eta(\bar{i}) \mid X_{i_{1}+s+s^{2}}, X_{i_{2}-s^{2}}, X_{i_{2}+s+s^{2}}\right\}-
\end{gathered}
$$




$$
\begin{gathered}
-\mathbf{E}\left\{\eta(\bar{i}) \mid X_{i_{1}-s^{2}}, X_{i_{1}+s+s^{2}}, X_{i_{2}-s^{2}}, X_{i_{2}+s+s^{2}}\right\} \mid \leqslant \\
\leqslant 4 \rho^{s-1}(1-\rho) e^{-c_{2}\left(s^{2}-2 s\right)} \sum_{k=1}^{N} \pi_{k}^{\prime} z_{k}^{-1} \sum_{l=1}^{N} \pi_{l}^{2} z_{l} .
\end{gathered}
$$

Поэтому из (3) и оценки $\left|J_{s, n}\right|<n^{2} / 2$ следует, что

$$
\left.\sum_{\bar{i} \in J_{s, n}} \mathbf{E} \mid \mathbf{E} \eta(\bar{i})-\mathbf{E}\left\{\eta(\bar{i}) \mid V_{\bar{i}}\right\}\right) \mid=O\left(\Lambda_{s, n} e^{-c s^{2}}\right)
$$

при любом $0<c<c_{4}$.

Подставляя оценки (12), (14) - (20) в (13) и используя условие $s^{3}<n$, убеждаемся в том, что при $s, n \rightarrow \infty$

$$
\begin{gathered}
\rho\left(\mathcal{L}\left(\delta_{n}(s)\right), \operatorname{Po}\left(\mathbf{E} \delta_{n}(s)\right)\right)=O\left(\frac{s^{2}}{n} \mathbf{E} \delta_{n}(s)\right)+ \\
+O\left(s^{3} n p^{s}+\frac{1-e^{-\mathbf{E} \delta_{n}(s)}}{\mathbf{E} \delta_{n}(s)}\left(s n p^{s}\right)^{2}+\min \left(\mathbf{E} \delta_{n}(s), \sqrt{\mathbf{E} \delta_{n}(s)}\right) e^{-c s^{2}}\right)= \\
=O\left(s^{2} n^{-1} \mathbf{E} \delta_{n}(s)+s^{3} n p^{s}+\min \left(\mathbf{E} \delta_{n}(s), \sqrt{\mathbf{E} \delta_{n}(s)}\right) e^{-c s^{2}}\right)
\end{gathered}
$$

при любом $0<c<c_{4}$.

3. Из соотношений $(10),(21)$ и неравенства треугольника следует, что

$$
\rho\left(\mathcal{L}\left(\tilde{\xi}_{n}(s)\right), \operatorname{Po}\left(\mathbf{E} \delta_{n}(s)\right)\right)=O\left(\rho\left(\mathcal{L}\left(\delta_{n}(s)\right), \operatorname{Po}\left(\mathbf{E} \delta_{n}(s)\right)\right)\right) .
$$

Из (22) и (21) следует (8). Теорема 5 доказана.

Доказательство теоремы 1. Нетрудно убедиться, что в предположениях теорем 1 и 2 при $s, n \rightarrow \infty$ выполнены соотношения

$$
s=O(\ln n), \frac{1}{n}=O\left(e^{-c_{3} s}\right), \quad s^{3} n p^{s}=O\left(s^{3}\left(\frac{p^{2}}{\rho}\right)^{s / 2}\right)=O\left(e^{-c s}\right)
$$

для любого $c, 0<c<c_{2}=\frac{1}{2} \ln \left(\frac{\rho}{p^{2}}\right)$. Кроме этого,

$$
e^{-c s^{2}}=O\left(e^{-d s}\right) \quad \forall c, d>0 .
$$

Из (8), (23), (24) и условий теоремы 1 следует, что

$$
\rho\left(\mathcal{L}\left(\tilde{\xi}_{n}(s)\right), \operatorname{Po}\left(\mathbf{E} \delta_{n}(s)\right)\right)=O\left(e^{-c s}\right)
$$

при любом $0<c<\min \left\{c_{2}, c_{3}\right\}$.

Далее воспользуемся теоремой 1.С и замечанием 1.1.4 книги [12], согласно которым с учетом условий теоремы 1 и оценки (12)

$$
\begin{gathered}
\rho\left(\operatorname{Po}\left(\mathbf{E} \delta_{n}(s)\right), \operatorname{Po}(\lambda)\right) \leqslant \min \left\{1, \frac{1}{\sqrt{\mathbf{E} \delta_{n}(s)}}\right\}\left|\mathbf{E} \delta_{n}(s)-\lambda\right|= \\
=O\left(\left|\mathbf{E} \delta_{n}(s)-\Lambda_{s, n}\right|+\left|\Lambda_{s, n}-\lambda\right|\right)= \\
=O\left(\frac{s^{3}}{n}+e^{-c_{1} s}+\left|\Lambda_{s, n}-\lambda\right|\right)=O\left(e^{-c s}+\left|\Lambda_{s, n}-\lambda\right|\right)
\end{gathered}
$$

при любом $0<c<\min \left\{1, c_{1}\right\}$. Из (25) и (26) следует (4). Теорема 1 доказана. 
Доказательство теоремы 2. В условиях теоремы 2 из (12) вытекает соотношение $\mathbf{E} \delta_{n}(s) \rightarrow \infty$, а потому из (8) следует равенство

$$
\rho\left(\mathcal{L}\left(\tilde{\xi}_{n}(s)\right), \operatorname{Po}\left(\mathbf{E} \delta_{n}(s)\right)\right)=O\left(s^{2} n^{-1} \mathbf{E} \delta_{n}(s)+s^{3} n p^{s}+\sqrt{\mathbf{E} \delta_{n}(s)} e^{-c s^{2}}\right)
$$

при любом $0<c<c_{4}$. Из этого соотношения, (23), (24) и условия (5) следует, что

$$
\rho\left(\mathcal{L}\left(\tilde{\xi}_{n}(s)\right), \operatorname{Po}\left(\mathbf{E} \delta_{n}(s)\right)\right) \rightarrow 0 .
$$

Пусть $F_{s, n}-$ функция распределения случайной величины $\Lambda_{s, n}^{-1 / 2}\left(\tilde{\xi}_{n}(s)-\mathbf{E} \tilde{\xi}_{n}(s)\right)$, a $G_{s, n}-$ функция распределения случайной величины $\left(\mathbf{E} \delta_{n}(s)\right)^{-1 / 2}\left(\zeta-\mathbf{E} \delta_{n}(s)\right)$, где $\zeta$ имеет распределение $\operatorname{Po}\left(\mathbf{E} \delta_{n}(s)\right)$. Используя (12) и $(23)$, получаем

$$
\frac{\tilde{\xi}_{n}(s)-\mathbf{E} \tilde{\xi}_{n}(s)}{\sqrt{\Lambda_{s, n}}}=\left(\frac{\tilde{\xi}_{n}(s)-\mathbf{E} \delta_{n}(s)}{\sqrt{\mathbf{E} \delta_{n}(s)}}+\frac{\mathbf{E} \delta_{n}(s)-\mathbf{E} \tilde{\xi}_{n}(s)}{\sqrt{\mathbf{E} \delta_{n}(s)}}\right)(1+o(1)) .
$$

Из (10), (23) и условия (5) следует, что

$$
\frac{\mathbf{E} \delta_{n}(s)-\mathbf{E} \tilde{\xi}_{n}(s)}{\sqrt{\mathbf{E} \delta_{n}(s)}} \rightarrow 0 .
$$

В свою очередь, из (27) следует, что

$$
\sup _{x}\left|\mathbf{P}\left\{\frac{\tilde{\xi}_{n}(s)-\mathbf{E} \delta_{n}(s)}{\sqrt{\mathbf{E} \delta_{n}(s)}}<x\right\}-G_{s, n}(x)\right| \rightarrow 0 .
$$

Все вместе это означает, что

$$
\sup _{x}\left|F_{s, n}(x)-G_{s, n}(x)\right| \rightarrow 0 .
$$

Осталось воспользоваться тем, что функция $G_{s, n}(x)$ центрированного и нормированного естественным образом пуассоновского распределения с параметром $\mathbf{E} \delta_{n}(s)$ при $\mathbf{E} \delta_{n}(s) \rightarrow \infty$ сходится к стандартной нормальной функции распределения. Теорема 2 доказана.

Доказательство теоремы 3. Следуем схеме доказательства теоремы 2 работы [1]. Положим $n(x)=\left[x R^{-1}\right]$. Согласно (3) при $s \rightarrow \infty$ выполнено соотношение $R=$ $O\left(\rho^{s / 2}\right)$. Поэтому

$$
\Lambda_{s, n}=\frac{n^{2}(x)}{2} R^{2}=\frac{1}{2}\left(\left[\frac{x}{R}\right] R\right)^{2}=\frac{x^{2}}{2}+O\left(\rho^{s / 2}\right) .
$$

Из (29) и теоремы 1 следует, что

$$
\mathbf{P}\{\tau(s) R>x\}=\mathbf{P}\left\{\tilde{\xi}_{n(x)}(s)=0\right\}=e^{-x^{2} / 2}+O\left(e^{-c s}+\left|\Lambda_{s, n}-x^{2} / 2\right|\right),
$$

для любого $c, 0<c<\min \left\{1, c_{1}, c_{2}, c_{3}\right\}$. Из (30) и (29) следует (6). Теорема 3 доказана.

Доказательство теоремы 4 аналогично доказательству п. б) теоремы 3 работы [1], и мы его не приводим.

Авторы признательны А. М. Зубкову и С. И. Чечете за полезные замечания. 


\section{Список литературы}

1. Зубков А.М., Михайлов В.Г., "Предельные распределения случайных величин, связанных с длинными повторениями в последовательности независимых испытаний", Теория вероятн. и ее примен., 19:1 (1974), 173-181.

2. Беляев П.Ф., "О совместном распределении частот длинных $s$-цепочек в мультиномиальной схеме с равновероятными исходами”, Теория вероятн. и примен., 14:3 (1969), 540-546.

3. Зубков А.М., Михайлов В.Г., "О повторениях $s$-цепочек в последовательности независимых величин", Теория вероятн. и примен., 24:2 (1979), 267-279.

4. Михайлов В.Г., Шойтов А.М., "Структурная эквивалентность $s$-цепочек в случайных дискретных последовательностях", Дискретная математика, 15:4 (2003), 7-34.

5. Hansen N. R., "Local alignment of Markov chains", Ann. Appl. Probab., 16:3 (2006), $1262-1296$.

6. Chryssaphinou O., Vaggelatou E., "Compound poisson approximation for multiple runs in a Markov chain", Ann. Inst. Statist. Math., 54:2 (2002), 411-424.

7. Беляев П.Ф., "K вопросу о совместном распределении частот $s$-цепочек в сложных цепях Маркова с большим числом состояний", Теория вероятн. и примен., 14:2 (1969), 333-339.

8. Беляев П.Ф., “О совместном распределении частот исходов в цепях Маркова с большим числом состояний", Теория вероятн. и примен., 22: 3 (1977), 534-545.

9. Зубков А.М., "Неравенства для вероятностей переходов с запрещениями и их применения", Матем. сб., 109(151):4(8) (1979), 491-532.

10. Гантмахер Ф.Р., Теория матрии. 5-е изд., М.: ФИЗМАТЛИТ, 2004, 576 с.

11. Шойтов А.М., "Пуассоновское приближение для числа повторений значений дискретной функции от цепочек", Дискретная математика, 17:2 (2005), 56-69.

12. Barbour A.D., Holst L., Janson S., Poisson Approximation, Oxford: Oxford University Press, 1992, 277 pp. 